\title{
On the Inverse Gaussian Modeling of Rainfall Rate and Slant Path and Terrestrial Links Rain Attenuation
}

\author{
Charilaos I. Kourogiorgas, Athanasios D. \\ Panagopoulos, Ioannis D. Kanellopoulos \\ School of Electrical and Computer Engineering \\ National Technical University of Athens \\ Athens, Greece \\ E-mail: harkour@mail.ntua.gr, thpanag@ece.ntua.gr, \\ ikanell@ece.ntua.gr
}

\author{
George K. Karagiannidis \\ Department of Electrical and Computer Engineering \\ Aristotle University of Thessaloniki \\ Thessaloniki, Greece \\ E-mail: geokarag@auth.gr
}

\begin{abstract}
The Inverse Gaussian (IG) distribution is examined for modeling the rainfall rate and slant path and terrestrial link rain attenuation. The long-term statistics of rain rate and rain attenuation are modeled using the IG distribution. The method is validated using the recommendation of International Telecommunication Union (ITU) recommendation ITU-R. P. 837 and rain rate data from the ITU Study Group 3 database (DBSG3) database for the case of rain rate. For the modeling of rain attenuation, data which are derived from two databases of DBSG3, these of Earth-space links and line-of-sight terrestrial links are used for validating the model. The results are compared to the one using the lognormal distribution. It has been shown that IG distribution could be more appropriate for modeling rainfall rate and slant path and terrestrial link rain attenuation. Finally, some useful conclusions are derived and presented in this paper.
\end{abstract}

Keywords-component; rain, radio propagation, satellite communications, wireless communications.

\section{INTRODUCTION}

Large bandwidth for the modern wireless systems is required in order to provide users with high data rate communications. In addition to the demand of high data rate services, the congestion of conventional frequency bands lead the future wireless terrestrial and satellite communication systems to operate at frequency bands higher than $10 \mathrm{GHz}$. In [1] the concept of the future Terabit/s satellite is introduced, supporting that high data rates can be provided only at $\mathrm{Ka}$ and $\mathrm{Q} / \mathrm{V}$ frequency bands.

At these frequency bands, the propagated electromagnetic waves are mainly affected by the tropospheric phenomena. Clouds, precipitation and atmospheric gases attenuate the power of the signal and atmospheric turbulence causes scintillation to the amplitude of the propagated signal. Rain attenuation is the most severe cause of degradation of the signal [2].

Fade Mitigation Techniques (FMTs) must be integrated into the system in order to deal with the tropospheric propagation and improve the quality of the link [2]. FMTs usually require the long-term statistics of the rainfall rate and rain attenuation for predicting the annual statistics of rain attenuation.

Various models have been developed for the prediction of long-term statistics of rain attenuation in relation to the statistics of rainfall rate. In [3], [4] rainfall rate and rain attenuation are assumed to follow the lognormal distribution, while in [5] rain attenuation is modeled using the Weibull distribution. In [6], the point rainfall rate and rain attenuation are assumed to follow the gamma distribution. Moreover, in [7] a log-power law is chosen for the modeling of rainfall rate in order to derive statistics of rain attenuation.

In this paper, the Inverse Gaussian (IG) distribution [8] is proposed and investigated for the modeling of rainfall rate and slant-path and terrestrial links rain attenuation. Firstly, some statistical properties of the inverse Gaussian distribution are given. In Section 3, IG modeling of point rainfall rate is evaluated. The model is validated using rain rate data from ITU-R. P. 837-5 [9] and the DBSG3 database [10]. The results are also compared to these of the lognormal distribution. In Section 4, IG distribution is used for modeling slant path and terrestrial link rain attenuation, showing numerical results with rain attenuation data derived from Earth-space links and lineof-sight terrestrial links database. Finally, some useful conclusions are derived from this paper.

\section{INVERSE GAUSSIAN DISTRIBUTION}

A random variable $X>0$ is assumed to follow the IG distribution, if the PDF of $X$ is this of (1) [9], [11].

$$
f_{X}(x)=\sqrt{\frac{\lambda}{2 \pi}} x^{-3 / 2} \exp \left(-\frac{\lambda(x-\mu)^{2}}{2 \mu^{2} x}\right)
$$

where, $\lambda$ and $\mu$ are the scale parameter and the mean value of the distribution, both greater than 0 (positive values).

The $n$-th moment, for $n$ integer, of the random variable $X$ is:

$E\left[x^{n}\right]=\mu^{n} \sum_{k=0}^{n-1} \frac{(n-1+k) !}{k !(n-1-k) !}\left(2 \frac{\lambda}{\mu}\right)^{-k}$ 
where E[ ] denotes the expected value. Therefore, the mean value and the variance of the random variable are:

$m_{x}=E[x]=\mu$

$\sigma_{x}^{2}=E\left[x^{2}\right]-E[x]^{2}=\frac{\mu^{3}}{\lambda}$

The Complementary Cumulative Distribution Function $(\mathrm{CCDF})$ is given in (5).

$$
P\left[X \geq x_{t h}\right]=1-Q\left(\sqrt{\frac{\lambda}{x_{t h}}}\left(1-\frac{x_{t h}}{\mu}\right)\right)-e^{2 \frac{\lambda}{\mu}} Q\left(\sqrt{\frac{\lambda}{x_{t h}}}\left(1+\frac{x_{t h}}{\mu}\right)\right)
$$

where $\mathrm{Q}($ ) is the Gaussian Q-function and is related to the complementary error function:

$$
Q(x)=\frac{1}{\sqrt{2 \pi}} \int_{x}^{+\infty} e^{-\frac{t^{2}}{2}} d t=\frac{1}{2} \operatorname{erfc}\left(\frac{x}{\sqrt{2}}\right)
$$

\section{RAIINFALL RATE MODELLING}

As the models for the prediction of rain attenuation are based on the statistics of rainfall rate, the modeling of rain rate is very crucial for channel modeling. The CCDF of point rainfall rate is the product of the probability to rain $\left(P_{0}\right)$ and the conditional CCDF of rain rate given that it is raining, as this is shown in (7)

$$
P\left[R \geq R_{t h}\right]=P_{o} P\left[R \geq R_{t h} / R>0\right]
$$

The $P_{0}$ value can be derived from the recommendation ITUR. P. 837-5 for every place in the world [9]. In propagation community, it is well accepted and assumed that the conditional CCDF of rainfall rate follows the lognormal distribution. In this case, the CCDF is given in (8), where $m_{\ln R}$ and $s_{\ln R}$ are the mean value and the standard deviation of the natural logarithm of rainfall rate.

$$
P\left[R \geq R_{t h}\right]=\frac{P_{o}}{2} \operatorname{erfc}\left(\frac{\ln R_{t h}-m_{\ln R}}{\sqrt{2} s_{\ln R}}\right)
$$

In this paper, it is assumed that the conditional CCDF of rainfall rate is given by the CCDF of IG distribution. Therefore, the conditional CCDF of rain rate is:

$$
P\left[R \geq R_{h h} / R>0\right]=1-Q\left(\sqrt{\frac{\lambda_{R}}{R_{h h}}}\left(1-\frac{R_{h h}}{\mu_{R}}\right)\right)-e^{2^{\frac{\lambda_{R}}{\mu_{R}}}} Q\left(\sqrt{\frac{\lambda_{R}}{R_{h h}}}\left(1+\frac{R_{h h}}{\mu_{R}}\right)\right)
$$

For the validation of the assumption, the CCDF of rain rate derived from ITU-R. P. 837-5 and from DBSG3 database will be used for fitting (7) and (9) for the IG distribution and (8) for the lognormal distribution to data. This fitting process is a nonlinear regression process and it results to the computation of the parameters $\lambda_{R}$ and $\mu_{R}$ for the first case and $m_{l n R}$ and $s_{l n R}$ for the lognormal distribution. As it is mentioned before, for both cases, the $P_{0}$ value is derived from the recommendation ITU-R. P. 837-5.

The CCDF of rain rate was derived using ITU-R. P. 837 for four places. In Figure 1, the CCDF of rain rate is shown for Prague, Czech Republic (latitude $=50^{\circ}$, longitude $=14.46^{\circ}$ ). In Figures 2, 3 and 4 the CCDF of rain rate for Athens, Greece (latitude $=37.89^{\circ}$, longitude $=23.8^{\circ}$ ), Rio de Janeiro, Brazil (latitude $=-22.11^{\circ}, \quad$ longitude $=318.43^{\circ}$ ) and Tokyo, Japan (latitude $=35.55^{\circ}$, longitude $=139.68^{\circ}$ ) are depicted, respectively. From all these 4 Figures, it can be observed that the IG distribution models shows better accuracy for the modeling of the rainfall rate CCDF and especially for high rain rate values than lognormal distribution.

For Figures 5 and 6, the CCDF of rain rate is taken from DBSG3 database for two places. The first one is located at Austin of USA (latitude $=30.4^{\circ}$, longitude $=262.3^{\circ}$ ) and the second one at Yamaguchi of Japan (latitude $=34.1^{\circ}$, longitude $=131 \cdot 6^{\circ}$ ). In Figure 5, for the case of Austin the IG distribution behaves better than the lognormal distribution for all probability levels and for the case of Yamaguchi, both distributions model accurately the rain rate distribution.

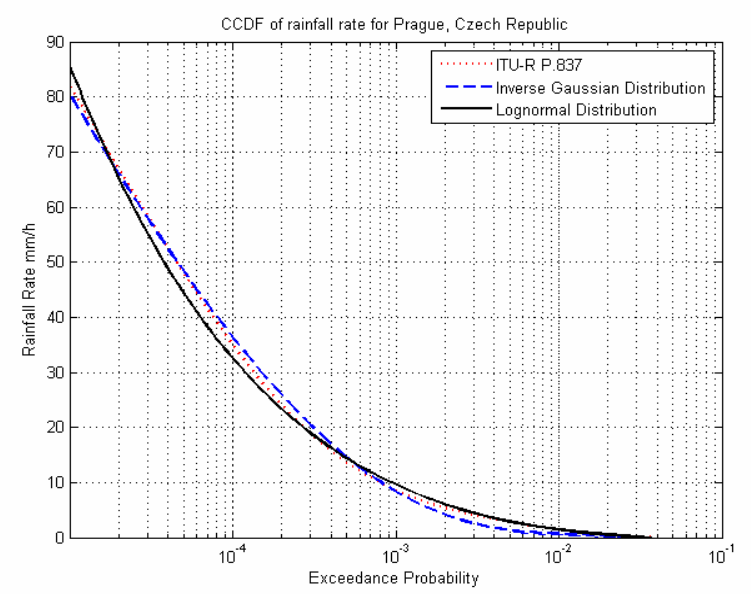

Figure 1. CCDF of rain rate for Prague, Czech Republic. The statistical parameters of IG distribution were computed $\lambda_{R}=0.0739 \mathrm{~mm} / \mathrm{h}$ and $\mu_{R}=1.0833 \mathrm{~mm} / \mathrm{h}$

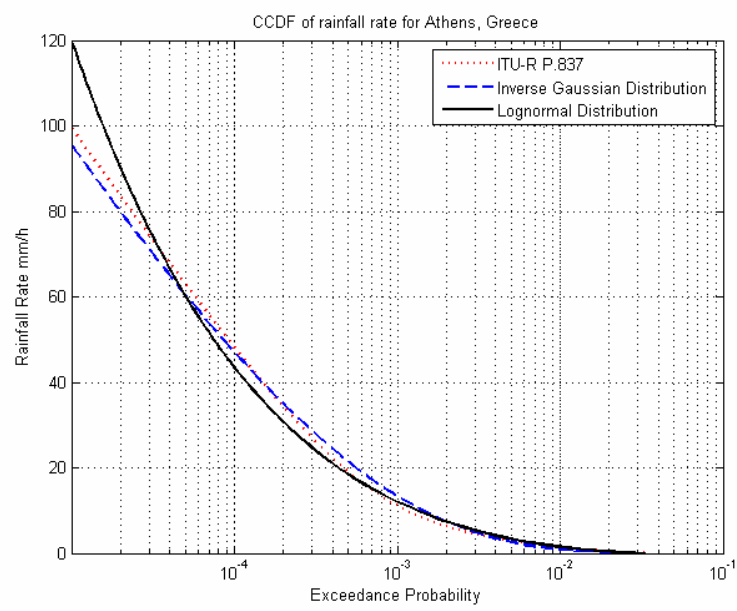

Figure 2. CCDF of rain rate for Athens, Greece. The statistical parameters of IG distribution were computed $\lambda_{R}=0.2420 \mathrm{~mm} / \mathrm{h}$ and $\mu_{R}=1.9980 \mathrm{~mm} / \mathrm{h}$ 


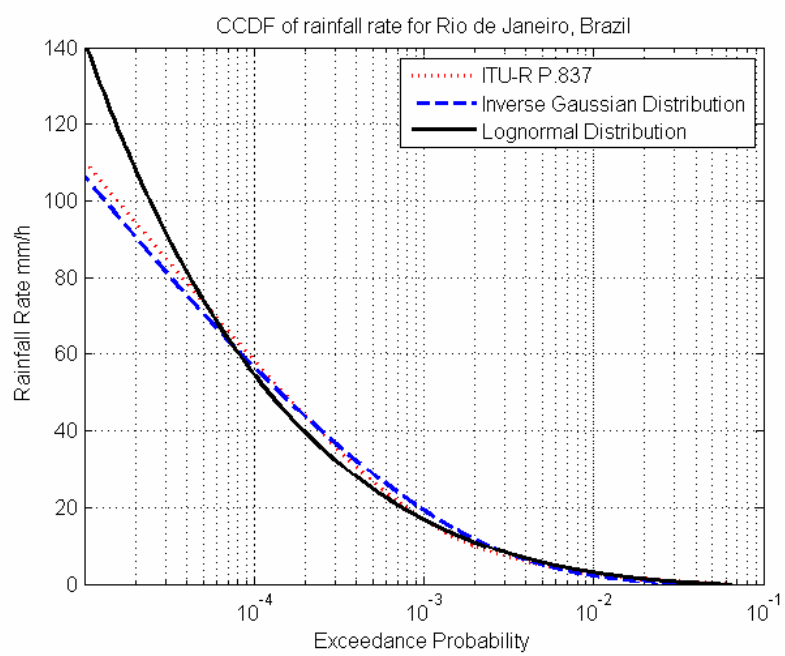

Figure 3. CCDF of rain rate for Rio de Janeiro, Brazil. The statistical parameters of IG distribution were computed $\lambda_{R}=0.1966 \mathrm{~mm} / \mathrm{h}$ and $\mu_{R}=1.7820$ $\mathrm{mm} / \mathrm{h}$

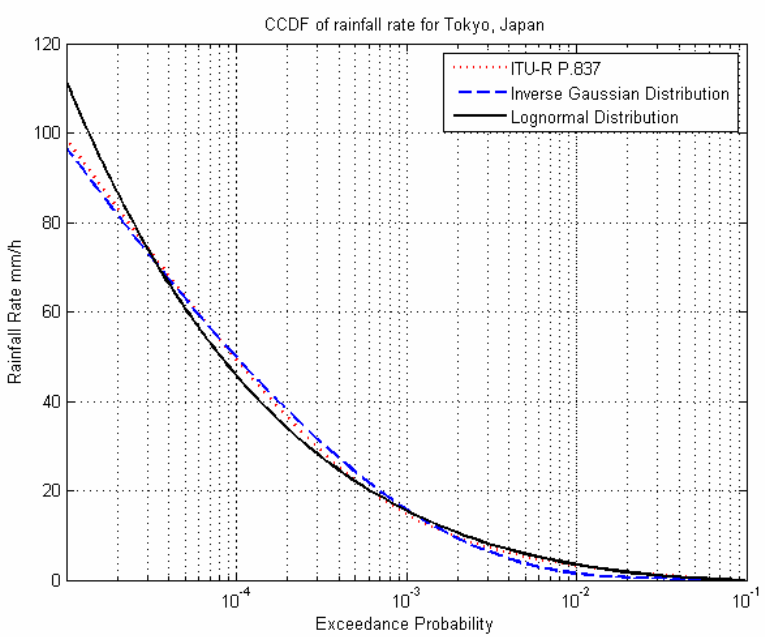

Figure 4. CCDF of rain rate for Tokyo, Japan. The statistical parameters of IG distribution were computed $\lambda_{R}=0.0573 \mathrm{~mm} / \mathrm{h}$ and $\mu_{R}=0.9426 \mathrm{~mm} / \mathrm{h}$

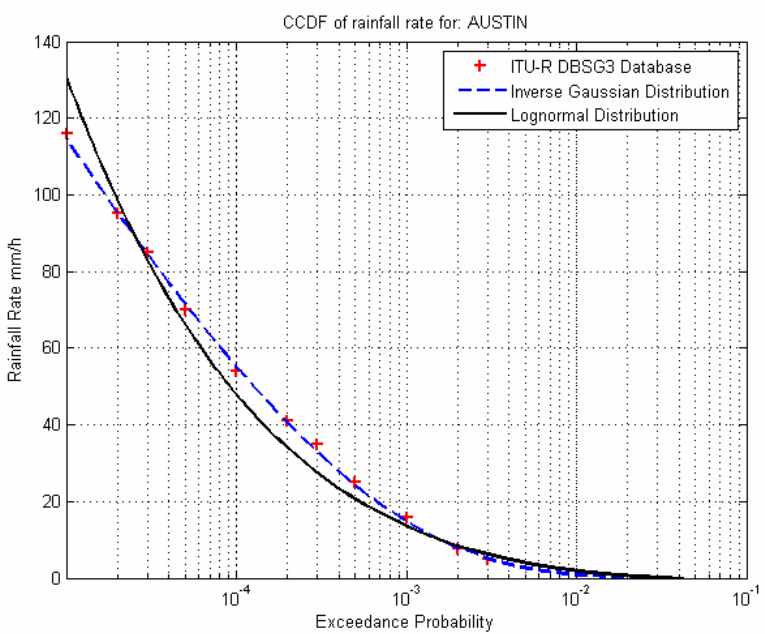

Figure 5. CCDF of rain rate for Austin, USA. The statistical parameters of IG distribution were computed $\lambda_{R}=0.1489 \mathrm{~mm} / \mathrm{h}$ and $\mu_{R}=1.7492 \mathrm{~mm} / \mathrm{h}$

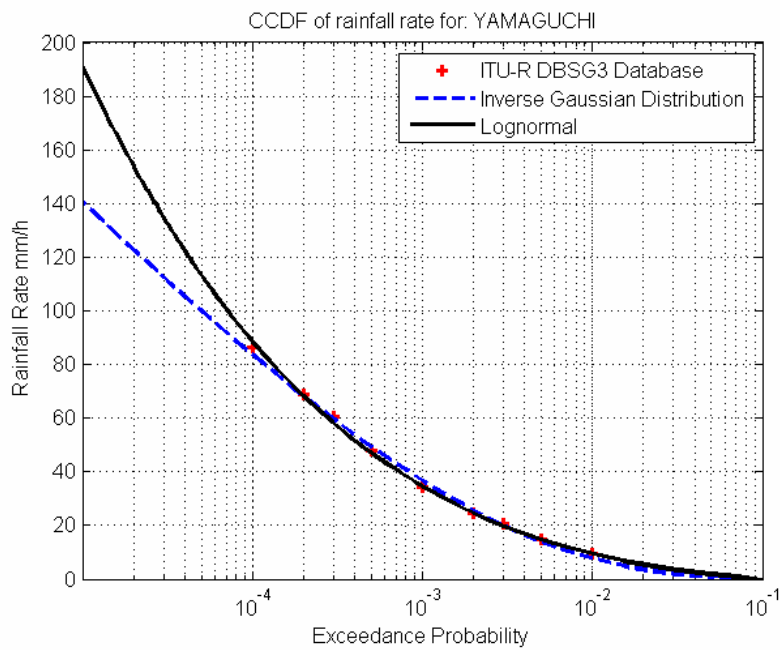

Figure 6. CCDF of rain rate for Yamaguchi, Japan. The statistical parameters of IG distribution were computed $\lambda_{R}=0.6414 \mathrm{~mm} / \mathrm{h}$ and $\mu_{R}=3.3231 \mathrm{~mm} / \mathrm{h}$

From the above Figures, it can be easily observed that the IG distribution can model accurately the long-term statistics of rain rate. The IG distribution behaves well for whatever the climatic region is, as temperate and tropical climates have bee used for the validation results. In comparison to the lognormal distribution, it can be referred that IG distribution is more accurate than lognormal, especially for low values of probability, in which lognormal distribution overestimates the rain rate. However, for high values of probability, close to $P_{0}$, the lognormal seems to be slightly better than IG distribution. In high time percentages, the IG distribution underestimates slightly the rain rate.

\section{RAIN ATtENUATION MODELING}

Rain is the most crucial factor of the attenuation of the signal and rain attenuation can be calculated from rainfall rate according to (10).

$A=\int_{0}^{L} a \cdot R^{b}(x) d x$

where $L$ is the effective path length, $R(x)$ is the rain rate at point $x$ and $a, b$ are the two coefficients of specific rain attenuation [12].

In the same way as in rain rate modeling the CCDF of rain attenuation is the product of the probability that rain attenuation exceeds $0 \mathrm{~dB}$ value $(P[A>0])$ multiplied by the conditional CCDF of rain attenuation, as this is shown in (11).

$$
P\left[A \geq A_{t h}\right]=P[\mathrm{~A}>0] \cdot P\left[A \geq A_{t h} / A>0\right]
$$

Since there is no accurate modeling of the $P[A>0]$ value in author's knowledge, the $P_{0}$ value derived from ITU-R. P. 837 for every place in the world will be used. Therefore, it is assumed that $P(A>0)=P(R>0)=P_{o}$.

The conditional CCDF is modeled using the IG distribution (12). The modeled rain attenuation will be validated using the DBSG3 database for Earth-space links and line-of-sight 
terrestrial links. The results will be compared with these of the lognormal distribution. As in the case of rainfall rate, the parameters of the IG and lognormal distributions will be derived through a nonlinear regression process, in which the theoretical CCDFs are fitted to rain attenuation data points.

$$
P\left[A \geq A_{t h} / A>0\right]=1-Q\left(\sqrt{\frac{\lambda_{A}}{A_{t h}}}\left(1-\frac{A_{t h}}{\mu_{A}}\right)\right)-e^{2 \frac{\lambda_{A}}{\mu_{A}}} Q\left(\sqrt{\frac{\lambda_{A}}{A_{t h}}}\left(1+\frac{A_{t h}}{\mu_{A}}\right)\right)
$$

\section{A. Earth-Space Links}

From the database of Earth-space links of DBSG3, three sites were selected. In Figure 7 the $\mathrm{CCDF}$ of rain attenuation for Clarksburg of USA (latitude $=39.2^{\circ}$, longitude $=282.7^{\circ}$ ) is shown. The other two sites are located in Spino d'Adda of Italy (latitude $=45.4^{\circ}$, longitude $=9.5^{\circ}$ ) and in UniTech Lae of Papua New Guinea (latitude $=-6.45^{\circ}$, longitude $=147^{\circ}$ ) and the distributions are shown in Figures 8 and 9 respectively.

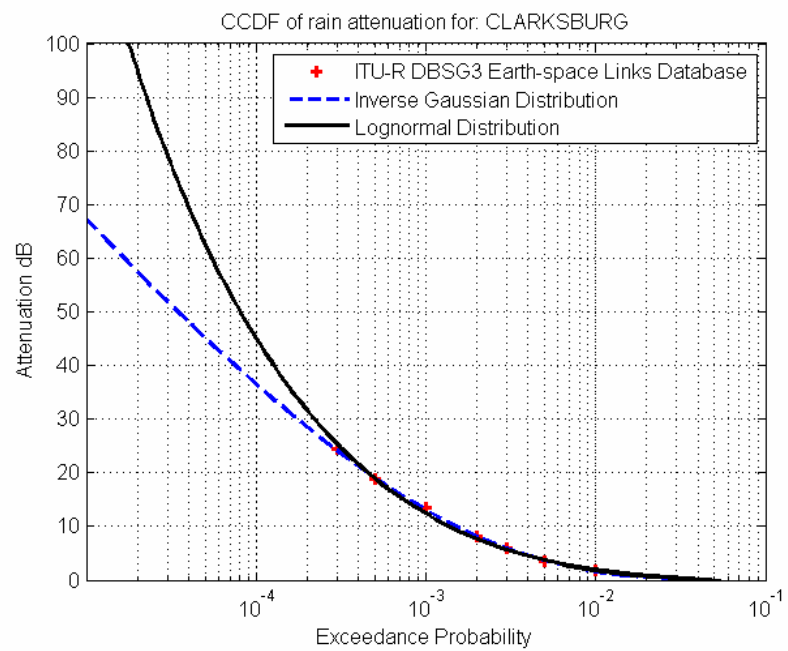

Figure 7. CCDF of rain attenuation for Clarksburg, USA. The statistical parameters of IG distribution were computed $\lambda_{A}=0.2209 \mathrm{~dB}$ and $\mu_{A}=1.4733 \mathrm{~dB}$

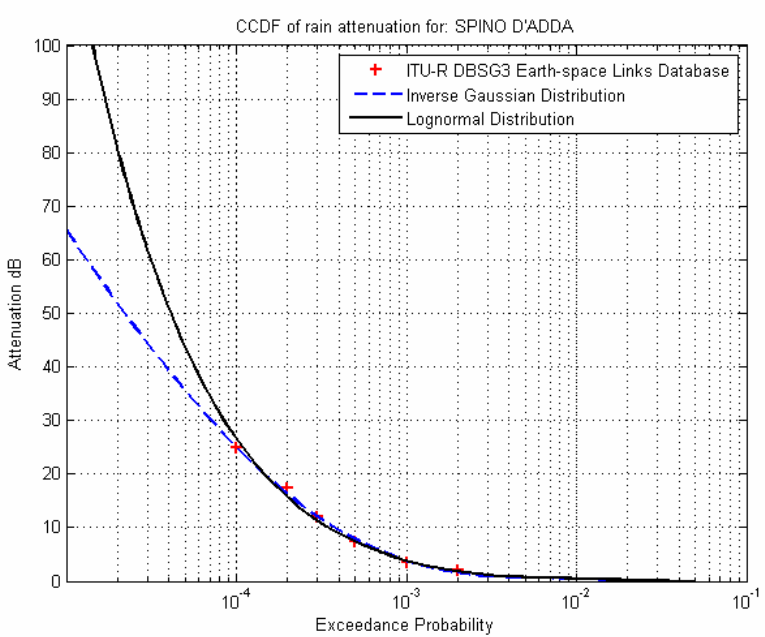

Figure 8. CCDF of rain attenuation for Spino d'Adda, Italy. The statistical parameters of IG distribution were computed $\lambda_{A}=0.0095 \mathrm{~dB}$ and $\mu_{A}=0.3942 \mathrm{~dB}$

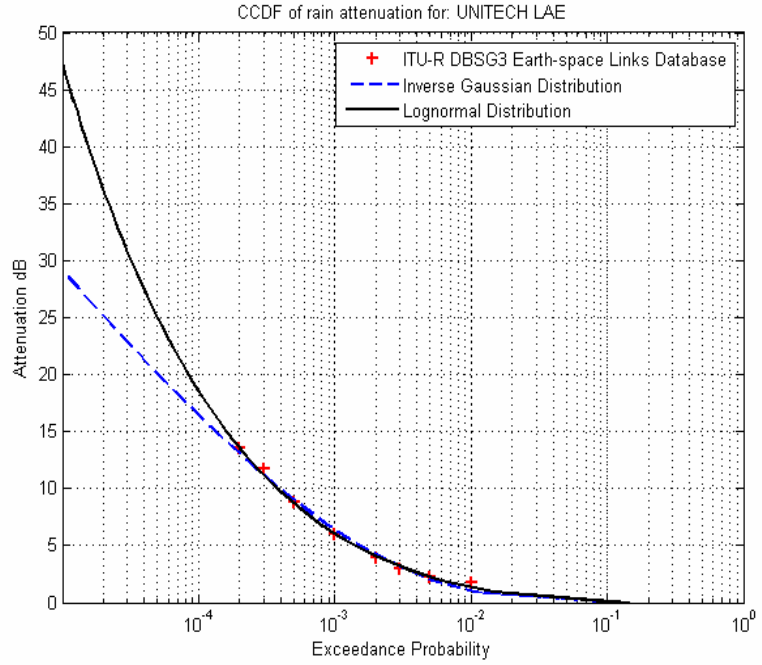

Figure 9. CCDF of rain attenuation for UniTech Lae, Papua New Guinea. The statistical parameters of IG distribution were computed $\lambda_{A}=0.0288 \mathrm{~dB}$ and $\mu_{A}=0.3385 \mathrm{~dB}$

From the above figures, it can be observed that the IG distribution models also accurately the conditional CCDF of rain attenuation for both temperate and tropical climates. The difference between the lognormal and the IG distribution is that for low probability values the IG distribution gives lower values of rain attenuation in comparison to the lognormal distribution. Furthermore, the difference between the two distributions for high percentages of probability in which lognormal distribution give higher values of rain rate than IG distribution is not observed in the case of rain attenuation for Earth-space links.

\section{B. Line-of-Sight Terrestrial Links}

The assumption of the IG distribution will be also examined with experiments derived from the line-of-sight terrestrial links DBSG3 database. For this purpose three experiments have been selected. For the first one, the receiver was located at Mendlesham of Great Britain (latitude $=52.26^{\circ}$, longitude $=1.1^{\circ}$ ) and the distributions are shown in figure 10 . For the other two experiments, the receivers were located at Darmstadt of Germany (latitude $=49.87^{\circ}$, longitude $=8.625^{\circ}$ ) and at Tokyo of Japan (latitude $=35.4^{\circ}$, longitude $=139.6^{\circ}$ ) and the distributions are shown in figures 11 and 12, respectively.

From these three Figures, it can be observed that also in the case of modeling of rain attenuation for line-of-sight terrestrial links, the IG distribution behaves really well, for all the three sites. Moreover, in comparison to the lognormal distribution, it can be referred that in case of Tokyo (Figure 12) the IG distributes lower values of rain attenuation for low levels of probability than lognormal distribution. This was also observed at the modeling of rain rate and modeling of rain attenuation for Earth-space links. At high percentages of probability, IG distribution gives lower values of rain attenuation than lognormal distribution, for all the three experiments. For the other levels of probability, both distributions have almost the same behavior, with IG distribution give more accurate results. 


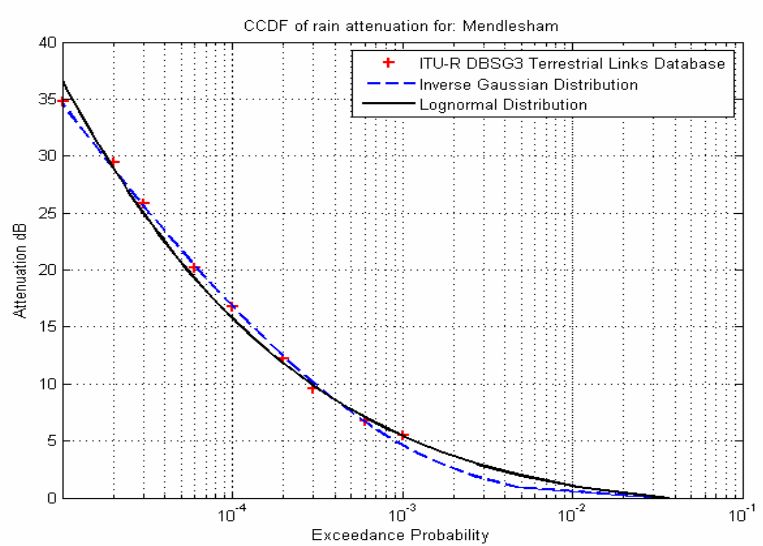

Figure 10. CCDF of rain attenuation for Mendlesham, Great Britain. The statistical parameters of IG distribution were computed $\lambda_{A}=0.064 \mathrm{~dB}$ and $\mu_{A}=0.6249 \mathrm{~dB}$

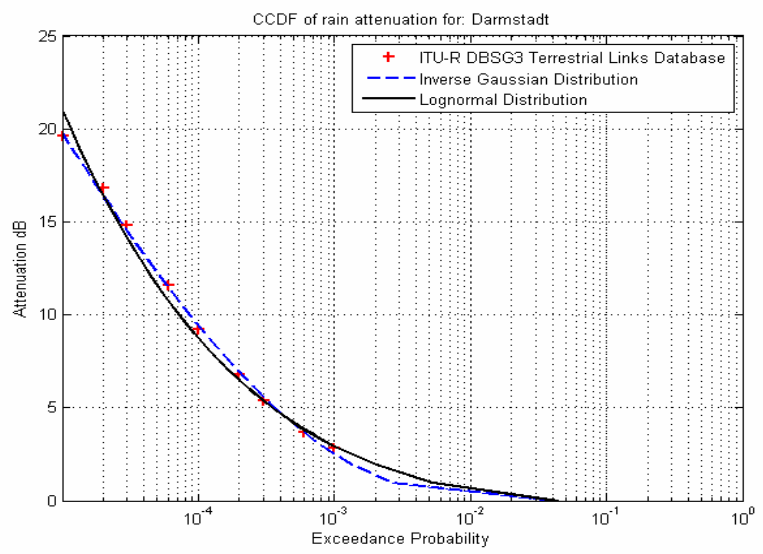

Figure 11. CCDF of rain attenuation for Darmstadt, Germany. The statistical parameters of IG distribution were computed $\lambda_{A}=0.0218 \mathrm{~dB}$ and $\mu_{A}=0.2789 \mathrm{~dB}$

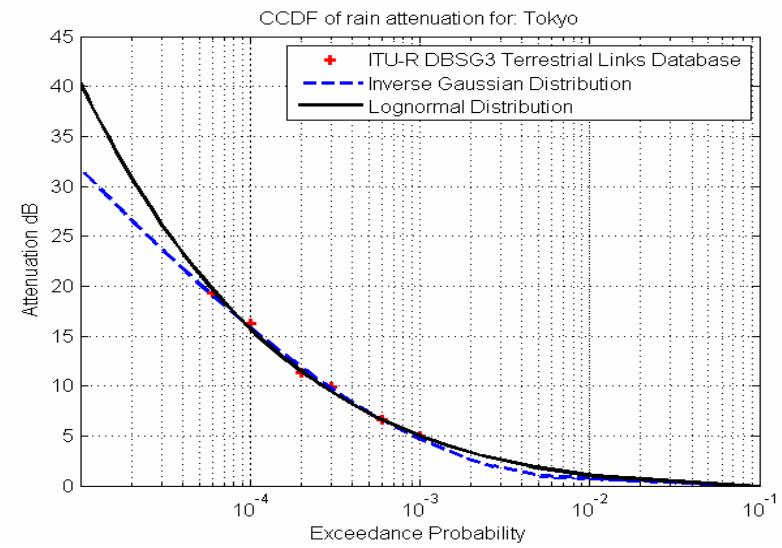

Figure 12. CCDF of rain attenuation for Tokyo, Japan. The statistical parameters of IG distribution were computed $\lambda_{A}=0.0125 \mathrm{~dB}$ and $\mu_{A}=0.2583 \mathrm{~dB}$

\section{CONCLUSIONS}

The IG distribution was investigated for modeling the long term (annual) statistics of rainfall rate and rain attenuation. The statistical parameters of the IG distribution were computed via a nonlinear regression with which the CCDF of IG distribution was fitted to the recommendation ITU-R. P.
837 and DBSG3 database for the modeling of rain rate and to rain attenuation data derived from Earth-space links and lineof-sight terrestrial links DBSG3 database. IG distribution models accurately the rain rate CCDF for both temperate and tropical climates. In comparison to the lognormal distribution, IG behaves better in low levels of probability as it distributes lower values of rain rate than lognormal distribution. However, in high levels of probability, lognormal distribution seems that models more accurately the CCDF of rain rate. For rain attenuation modeling, for both slant path and terrestrial link rain attenuation, IG distribution behaves very well for different climates. In comparison to the lognormal distribution, IG distribution gives lower values of rain attenuation for low percentages of probability, while for the other levels of probability both models give almost the same prediction results.

\section{ACKNOWLEDGMENT}

This research has been carried out in part under the framework of NTUA-THALES 'MIMOSA' project funded by EU and Greece.

\section{REFERENCES}

[1] P. Thompson, B. Evans, L. Castanet, M. Bousquet, T. Mathiopoulos, "Concepts and Technologies for a Terabit/s Satellite", SPACOMM 2011, Budapest, Hungary.

[2] A. D. Panagopoulos, P.-D. M. Arapoglou, P. G. Cottis, "Satellite Communications at $\mathrm{Ku}, \mathrm{Ka}$ and $\mathrm{V}$ bands: Propagation Impairements and Mitigation Techniques", IEEE Communication Surveys and Tutorials, 2004.

[3] A. D. Panagopoulos, J. D. Kanellopoulos, "On the Rain Attenuation Dynamics: Spatial-Temporal Analysis of Rainfall-rate and Fade Duration Statistics", Int. J. of Sat. Comm. And Net., vol. 21, no. 6, pp.595-611, 2003.

[4] S. H. Lin, "A Method for Calculating Rain Attenuation Distributions in Microwave Paths", Bell Syst. Techn. J., vol. 54, no. 6, p.. 1051-1083, 1975.

[5] A. D. Panagopoulos, P.-D. M. Arapoglou, J. D. Kanellopoulos, P. G. Cottis, "Long-Term Rain Attenuation Probability and Site Diversity Gain Predicition Formulas, IEEE Trans. On Antennas and Propagation, vol. 53 , no. 7, pp. 2307-2313, 2005.

[6] A. D. Panagopoulos, J. D. Kanellopoulos, "Adjacent Satellite Interference Effects as Applied to the Outage Performance of an EarthSpace System Located in a Heavy Rain Climatic Region", Annals of Telecommunications, no. 9-10, pp.925-9422, 2002.

[7] C. Capsoni, L. Luini, A. Paraboni, C. Riva, A. Martelucci, "A New Prediction Model of Rain Attenuation that Separately Accounts for Stratiform and Convective Rain", IEEE Trans. On Antennas and Propagation, vol. 57, no. 1, pp. 196-204, 2009.

[8] R. S. Chikkara, J. L. Folks, “ The Inverse Gaussian Distribution: Theory, Methodology and Applications”, Neq York: M. Dekker, 1989.

[9] ITU-R. P.837-5, "Characteristics of Precipitation for Propagation Modeling”, Geneva, 2007.

[10] (2010) ITU-R. Databank DBSG3, http://www.itu.int/publ/R-SOFT$\underline{\mathrm{SG} 3 / \mathrm{en}}$

[11] B. Seshardi, "The Inverse Gaussian Distribution", Clarendon Press, Oxford, 1993.

[12] ITU-R. 838-3, "Specific Attenuation Model for Rain for Use in Prediction Methods”, Geneva, 2005. 